\title{
Effects of Dietary Restriction on Cancer Development and Progression
}

\author{
Daniele Fanale, Rossella Maragliano, Alessandro Perez, and \\ Antonio Russo
}

\begin{abstract}
The effects of caloric restriction on tumor growth and progression are known for over a century. Indeed, fasting has been practiced for millennia, but just recently has emerged the protective role that it may exert toward cells. Fasting cycles are able to reprogram the cellular metabolism, by inducing protection against oxidative stress and prolonging cellular longevity. The reduction of calorie intake as well as short- or long-term fasting has been shown to protect against chronic and degenerative diseases, such as diabetes, cardiovascular pathologies, and cancer. In vitro and in vivo preclinical models showed that different restriction dietary regimens may be effective against cancer onset and progression, by enhancing therapy response and reducing its toxic side effects. Fasting-mediated beneficial effects seem to be due to the reduction of inflammatory response and downregulation of nutrient-related signaling pathways able to modulate cell proliferation and apoptosis. In this chapter, we will discuss the most significant studies present in literature regarding the molecular mechanisms by which dietary restriction may contribute to prevent cancer onset, reduce its progression, and positively affect the response to the treatments.
\end{abstract}

\section{Keywords}

Caloric restriction - Cancer - Cell proliferation - Diet - Dietary restriction • Fasting • Feeding • IGF-1 - Inflammatory response - Long-term starvation • Molecular pathways $\bullet$ Oxidative stress $\bullet$ Short-term starvation $\bullet$ Therapy response

Daniele Fanale and Rossella Maragliano are contributed equally to this work

D. Fanale $\bullet$ R. Maragliano $\bullet$ A. Perez $\bullet$ A. Russo $(\bowtie)$

Department of Surgical, Oncological and Oral Sciences, Section of Medical Oncology, University of Palermo, Palermo, Italy

e-mail: fandan@libero.it; rossellamaragliano88@gmail.com; ale-like@libero.it; antonio.

russo@usa.net 


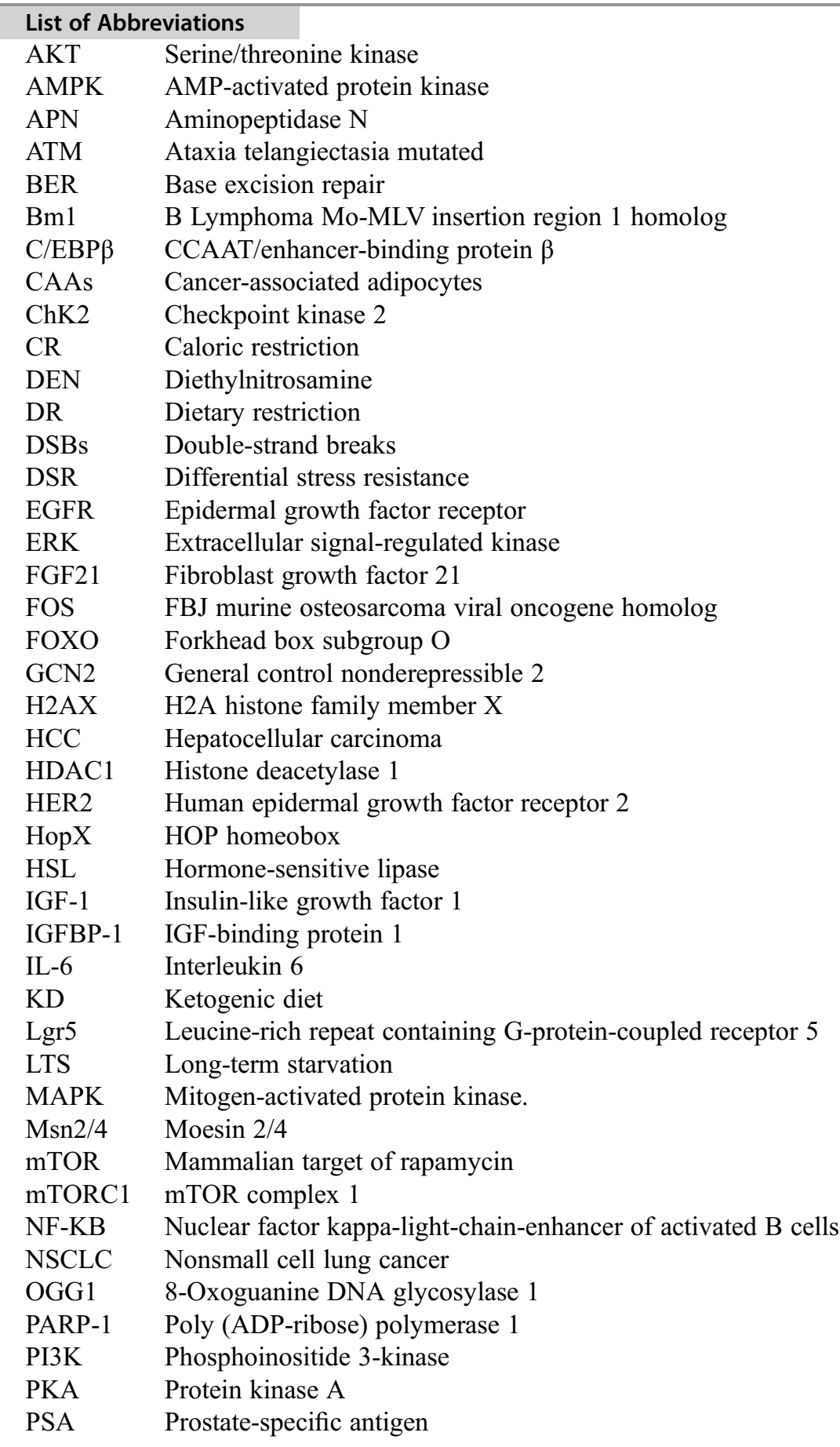




\begin{tabular}{ll}
\hline RAF & V-Raf-1 murine leukemia viral oncogene homolog \\
Ras & Rat sarcoma viral oncogene homolog \\
REV1 & DNA-directed polymerase \\
ROS & Reactive oxygen species \\
SIRT1 & Sirtuin 1 \\
SOD2 & Superoxide dismutase 2 \\
SSBs & Single-strand breaks \\
STS & Short-term starvation \\
TKIs & Tyrosine-kinase inhibitors \\
VEGF & Vascular endothelial growth factor
\end{tabular}

\section{Contents}

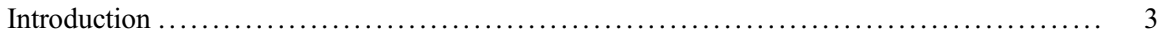

Molecular Pathways Involved in Dietary Restriction and Cancer-Related Events .......... 4

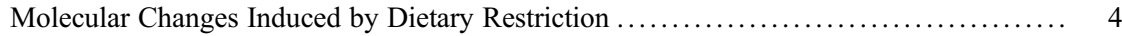

CR-Induced Changes in Tumor Microenvironment ........................... 4

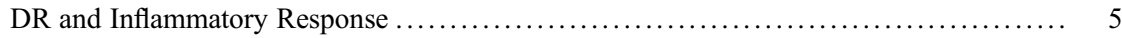

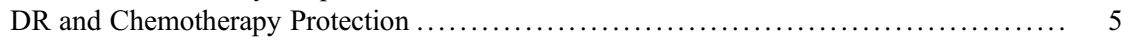

Correlations Between DR and IGF-1, Insulin, and Cancer ...................... 6

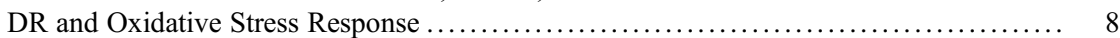

The Implication of Dietary Restriction in Cancer ............................... 10

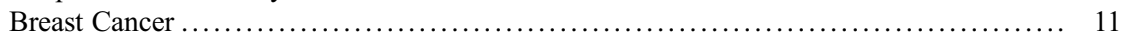

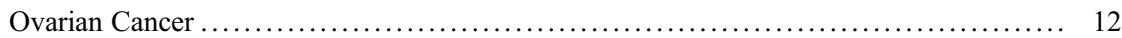

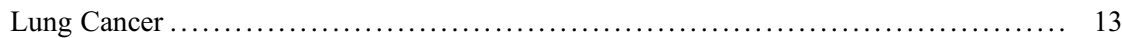

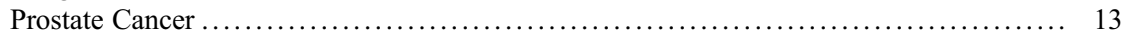

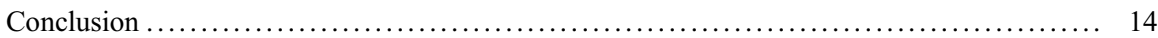

Policies and Protocols ...................................................... 14

Protocol for Maintaining Cancer Cells Under Short-Term Starvation Conditions ........ 14

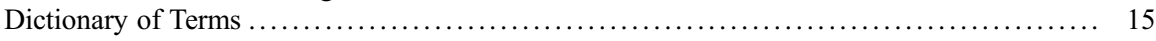

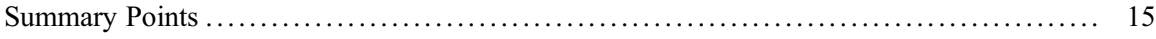

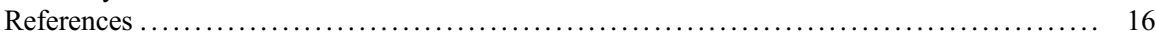

\section{Introduction}

In recent years, increasing evidences showed that several types of intermittent, chronic, or periodic dietary approaches, including short-term starvation (STS), long-term starvation (LTS or fasting), caloric restriction (CR), may exert a protective role against aging and other age-related pathologies as well as cancer in humans and numerous animal models (Lee and Longo 2016; Brandhorst and Longo 2016; Longo et al. 2015; Trepanowski et al. 2011). Interestingly, these dietary restriction (DR) regimens showed significant anticancer effects mostly in preclinical models, suggesting the possibility of using these methods to increase lifespan and improve therapy response in cancer patients. However, prolonged fasting periods could impair the patient health conditions already unfavorable due to physiological weight loss (Cleary and Grossmann 2011; Lluch et al. 2014). For this reason, STS (or intermittent fasting), consisting of the lack of food intake for a short time, appears to be the most suitable approach for cancer patients, although there are conflicting 
opinions about it. STS aims to slow down growth of tumor, by restricting temporarily its exposure to different nutrients, including glucose, and generating protective effects against cancer (Robertson and Mitchell 2013; Anton and Leeuwenburgh 2013). Conversely, LTS consists of a prolonged food deprivation, resulting in adaptive cellular responses able to decrease inflammatory processes and oxidative stress, enhance energy metabolism, and strengthen cell protection (Longo and Mattson 2014). For example, a serum starvation able to bring down basal cellular activity was applied to several in vitro models, in order to study molecular mechanisms underlying apoptosis, cellular stress response, and autophagy (Pirkmajer and Chibalin 2011). Finally, CR is defined as the reduction in calorie intake aimed to inhibit tumorigenesis and prevent other diseases, including diabetes and cardiovascular pathologies, by inducing an improved insulin sensitivity and reducing the oxidative damage and metabolic rate (Lv et al. 2014; Lefevre et al. 2009).

This chapter aims to provide an overview of the most recent studies present in literature concerning the molecular mechanisms by which dietary restriction may contribute to prevent cancer onset, slow down its progression, and positively affect the response to anticancer therapies, also suggesting a close correlation between diet and reduction of treatment-induced side effects.

\section{Molecular Pathways Involved in Dietary Restriction and Cancer- Related Events}

\section{Molecular Changes Induced by Dietary Restriction}

Nowadays, the link between cancer and metabolism is becoming increasingly evident (Longo and Mattson 2014; Brandhorst et al. 2017). It is clear that beneficial effects mediated by fasting, in particular by CR, do not involve a single gene, a pathway or a unique molecular mechanism. The benefits are due to the negative regulation of nutrient-signaling pathways, including insulin-like growth factor 1 (IGF-1) pathway and its effector extracellular signal-regulated kinase (ERK), mitogen-activated protein kinase (MAPK), and phosphoinositide 3-kinase (PI3K), which are known to modulate important proliferation pathways (Cangemi et al. 2016). Furthermore, it is well known that genomic instability is a distinctive feature of cancer, and CR tumor response seems to play a key role for the maintenance of genomic integrity (Robertson and Mitchell 2013; Duan et al. 2017). Due to these evidences, new metabolic approaches are being sought today for anticancer treatment. CR, also used in combination with the conventional chemotherapies, has allowed to obtain good results in animal models (Klement and Fink 2016).

\section{CR-Induced Changes in Tumor Microenvironment}

The most recent data in literature showed a correlation between aging and neoplastic diseases. It has been observed that aging promotes neoplastic cell growth and proliferation through surrounding microenvironment alterations. This process, 
named "adaptive oncogenesis," is determined by tissue decline caused by age and oncogenic cell alterations (Cadoni et al. 2017). Changes in age-associated tissue microenvironment seem to play an important role in cancer and cancer-related diseases. Although the mechanisms responsible for delays in aging and carcinogenesis have not been fully identified, CR is today the only known nongenetic approach able to extend organism life. Nutrient-sensing pathways play a pivotal role in cellular response to $\mathrm{CR}$ probably because these regulatory processes are responsible for maintaining a microenvironment that promotes aging and carcinogenicity (Cadoni et al. 2017).

The deacetylase SIRT1 is a protein implicated in regulation effects downstream of CR, in both human and murine models (Cohen et al. 2004). The SIRT1 levels are low in senescent cells probably due to the formation of the $\mathrm{C} / \mathrm{EBP} \beta$ complex and HDAC1, which bind and inhibit SIRT1 promoter. Several studies showed that long-term $\mathrm{CR}$ is able to block the formation of the C/EBP $\beta$ and HDAC1 inhibitory complex, restoring the functionality of SIRT1 promoter in murine liver cells (Jin et al. 2011).

The SIRT1-activated pathway is also involved in the regulation of forkhead box subgroup O (FOXO) protein, which is deacetylated by SIRT1 in response to oxidative stress. The FOXO1 levels seem to be increased in rat liver cells during long-term CR. This suggests that both SIRT1 and FOXO1 have a modulating role in long-term $\mathrm{CR}$ and are responsible for creating a microenvironment that delays aging and prevent cancer (Yamaza et al. 2010).

\section{DR and Inflammatory Response}

Several studies showed that DR also plays a role in modulating inflammatory response. Liver cells of diethylnitrosamine (DEN)-induced HCC mice models submitted to DR showed a reduction in levels of NF-kB, a mediator of inflammation associated with cell proliferation and cancer (Duan et al. 2017). A decrease in levels of cytokines and inflammatory chemokines was observed in murine liver, kidney, and spleen tissues (Chiba and Ezaki 2010). Also, mice under 4 weeks DR condition display a reduction of proinflammatory gene expression and an increase in antiinflammatory gene expression (Robertson and Mitchell 2013; Fig. 1).

\section{DR and Chemotherapy Protection}

Proliferation pathways regulated by Ras and AKT are almost always constitutively activated in cancer cells. Cells dramatically reduce the cell division number and become more resistant to stress in response to poor nutrition conditions, such as fasting or DR. This occurs because DR inactivates nutrient-sensing signaling pathways (Brandhorst et al. 2017). The link between cell proliferation, which depends on the nutrient-sensing pathways, and stress resistance is the basis of the protective effect that DR exerts on normal cells compared to tumor cells. This resistance is called differential stress resistance (DSR). In fact, tumor cells are unable to protect 


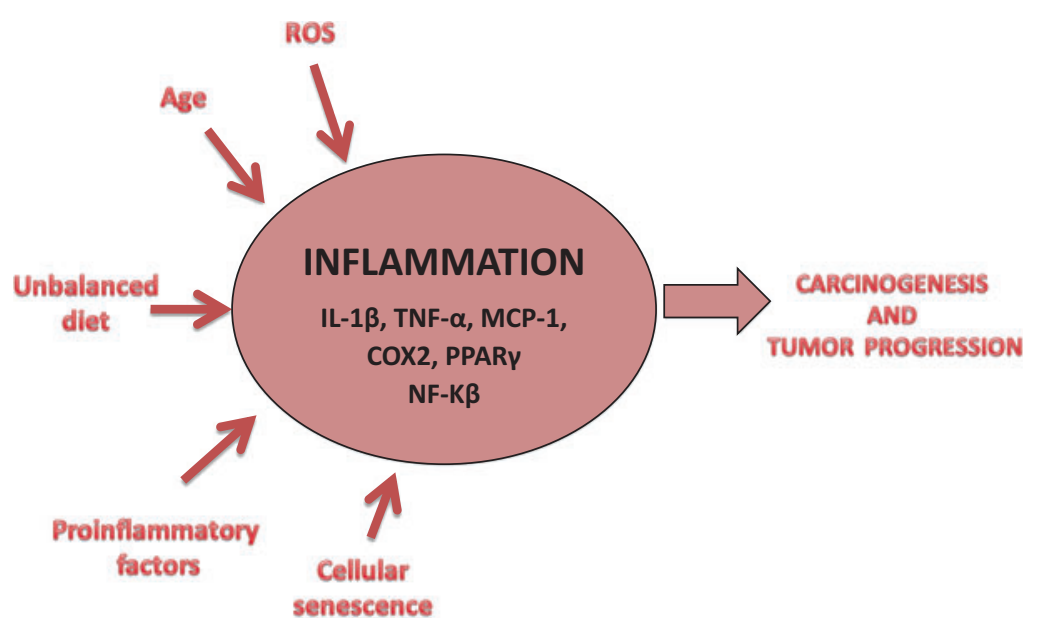

Fig. 1 Association between inflammation and cancer. Unbalance diet, age, cellular senescence, and accumulation of proinflammatory factors and ROS cause cell inflammation

themselves from stress, because oncogenes negatively regulate resistance genes (Brandhorst et al. 2017; Raffaghello et al. 2008). Moreover, several mutations accumulated in cancer cells make them less able to adapt to extreme environmental conditions created by fasting (Longo and Fontana 2010). Several studies showed that DR-induced DSR may be used to protect healthy cells from the toxic effects of chemotherapy (Brandhorst et al. 2017).

The fasting-induced protection has been shown also in in vivo experiments. CR protects mice from high dose etopoxide toxicity 4 , nausea and vomiting induced by doxorubicin, and irinotecan-induced weight loss (Raffaghello et al. 2008; Tinkum et al. 2015). It has also been found that IGF-1 gene deletion protects against chemotherapeutic toxicity of doxorubicin and cyclophosphamide (Brandhorst et al. 2017). Probably, the protective effect induced by DR and starvation is due to a change in microenvironment of the intestinal cryptic stem cells. Indeed, fasting before chemotherapy preserves the correct architecture and functioning of intestinal cells by maintaining the expression of genes such as Lgr5, Bm1, and HopX (Tinkum et al. 2015).

In other studies, it was observed that DR makes cancer cells susceptible to cisplatin-based chemotherapy effects through activation of the ATM/ChK2/p53 signaling pathway, which causes temporary loss of coordination between cell proliferation and growth stimulated by nutrients (Shi et al. 2012).

\section{Correlations Between DR and IGF-1, Insulin, and Cancer}

One of the nutrition-related pathways involved in carcinogenesis is the IGF-1 signaling, which affects both sensitivity to oxidative stress and DR. Insulin and IGF-1 play a pivotal role in controlling metabolism and growth in response to 
nutritional signals and nutritional state of cells (Shi et al. 2012). IGF-1 pathway regulates cell proliferation and differentiation, showing a tumorigenic effect through apoptosis inhibition (Ramsey et al. 2002; Prisco et al. 1999). Epidemiological studies highlighted the role that IGF-1 pathway plays in cancer pathology. Indeed, high serum IGF-1 concentration is associated with an increased risk of prostate, breast, and colon cancers (Renehan et al. 2004).

A study performed on murine xenograft models showed that deregulation of IGF1 and PI3K/AKT pathways results in DR resistance. IGF-1 recruits PI3K on cell membrane via binding to tyrosine kinase receptor, resulting in AKT activation. AKT, in turn, phosphorylates and activates downstream effectors that induce cell proliferation (Kalaany and Sabatini 2009).

FOXO1 protein is an effector downstream of IGF-1/AKT pathway, negatively regulated by AKT. This protein is able to modulate the expression of genes involved in oxidative metabolism, stress resistance, and longevity (Cangemi et al. 2016). It has been observed that DR-sensitive cells show a decrease in AKT cytoplasmic levels. This results in FOXO1 nuclear relocation and induction of the proapoptotic and antiproliferative gene transcription. In addition, in vivo studies on xenograft models demonstrated that DR-induced apoptosis increases in tumor cells that overexpress FOXO1. These results are consistent with the antitumorigenic effect of FOXO1 in DR conditions (Kalaany and Sabatini 2009).

A significant role is played by downstream effectors of the PI3K pathway, such as mTOR, AMPK, and SIRT1, which are probably related to cellular sensitivity to DR. Moreover, it has been observed that mutations constitutively activating PI3K protein are important for tumor sensitivity to DR. In fact, an increased sensitivity to DR is observed when PI3K levels decrease. This suggests that molecular analysis of PI3K mutational state could represent an interesting tool to identify DR resistance markers (Lee et al. 2012b; Fig. 2).

Several epidemiological studies showed that there is a strong correlation between increased adiposity and tumor risk (Lee et al. 2012b; Fanale et al. 2017; Wang et al. 2012; Toren et al. 2013; La Paglia et al. 2017). Adiposity is associated with an increase in insulin serum levels. Insulin, an anabolic hormone produced by pancreatic $\beta$-cells, exhibits mitogenic effects on many cell types, especially on preneoplastic cells. Furthermore, insulin increases IGF-1 activity, reducing synthesis and secretion of IGF-binding protein 1 (IGFBP-1) (Esposito et al. 2003). Hyperinsulinemia increases concentration of circulating sex hormones, in particular stimulating the production of androgens involved in the growth of different tumors. Several studies showed that DR counteracts metabolic anomalies associated with excessive adiposity, by reducing insulin levels, sex hormones, IGF-1, inflammatory cytokines, prostaglandins, and other various markers of oxidative stress and DNA damage (Esposito et al. 2003, Heilbronn et al. 2006; Fig. 3). According to the data of epidemiological studies carried out on dietary style of Western countries, DR associated with low protein intake has been shown to decrease serum IGF-1 levels in humans (Fontana et al. 2008; Giovannucci et al. 2003).

DR in combination with deprivation of essential amino acids triggers protective events for cells, by inducing a decrease in mTORC1 cellular levels and a concomitant increase in amino acid deprivation sensor (GCN2) (Brandhorst et al. 2017). 


\section{Dietary Restriction}

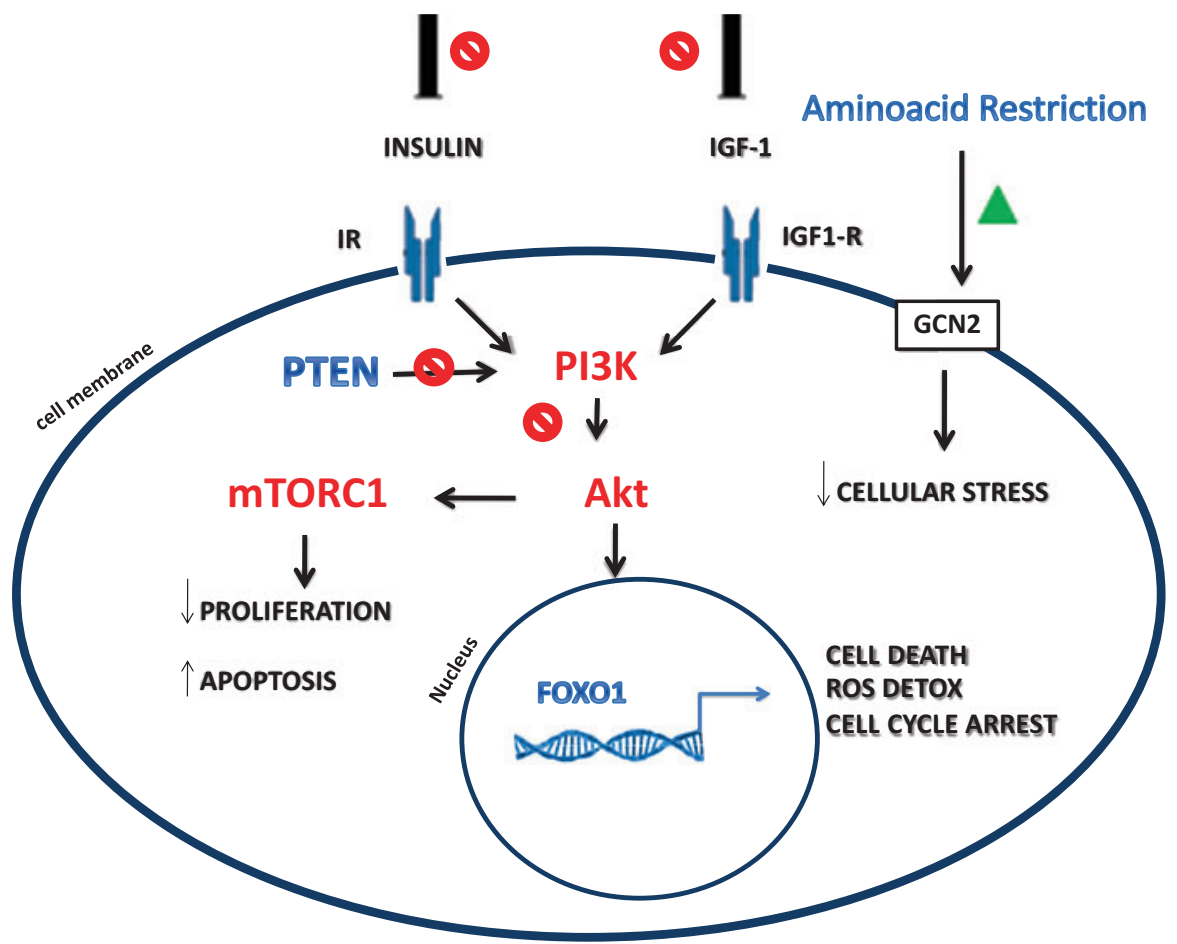

Fig. 2 Molecular pathways modulated by dietary restriction. Dietary restriction decreases the circulating levels of insulin and IGF-1, resulting in inhibition of PI3K/AKT pathway, and leading to increased apoptosis and decreased proliferation

Although there is still no data regarding the effect of fasting in preventing cancer in humans, the most likely hypothesis is that the effect of DR on IGF-1 levels could generate a protective environment for healthy cells and an adverse environment for tumor cell growth (Longo and Mattson 2014). Another protein restriction marker is FGF21, the fibroblast growth factor, regulated by PPAR $\alpha$ whose plasma levels increase during DR associated with protein restriction. In summary, DR has effects on FGF21, IGF-1, and mTOR activity, which are probably linked to carcinogenesis (Klement and Fink 2016).

\section{DR and Oxidative Stress Response}

Many studies indicated that the increase of antioxidant factors in tumor cells is mediated by threonine tyrosine kinase Rim15 and transcription factors Msn2/4 and Gis1, which regulate several genes, including mitochondrial SOD2, implicated in 


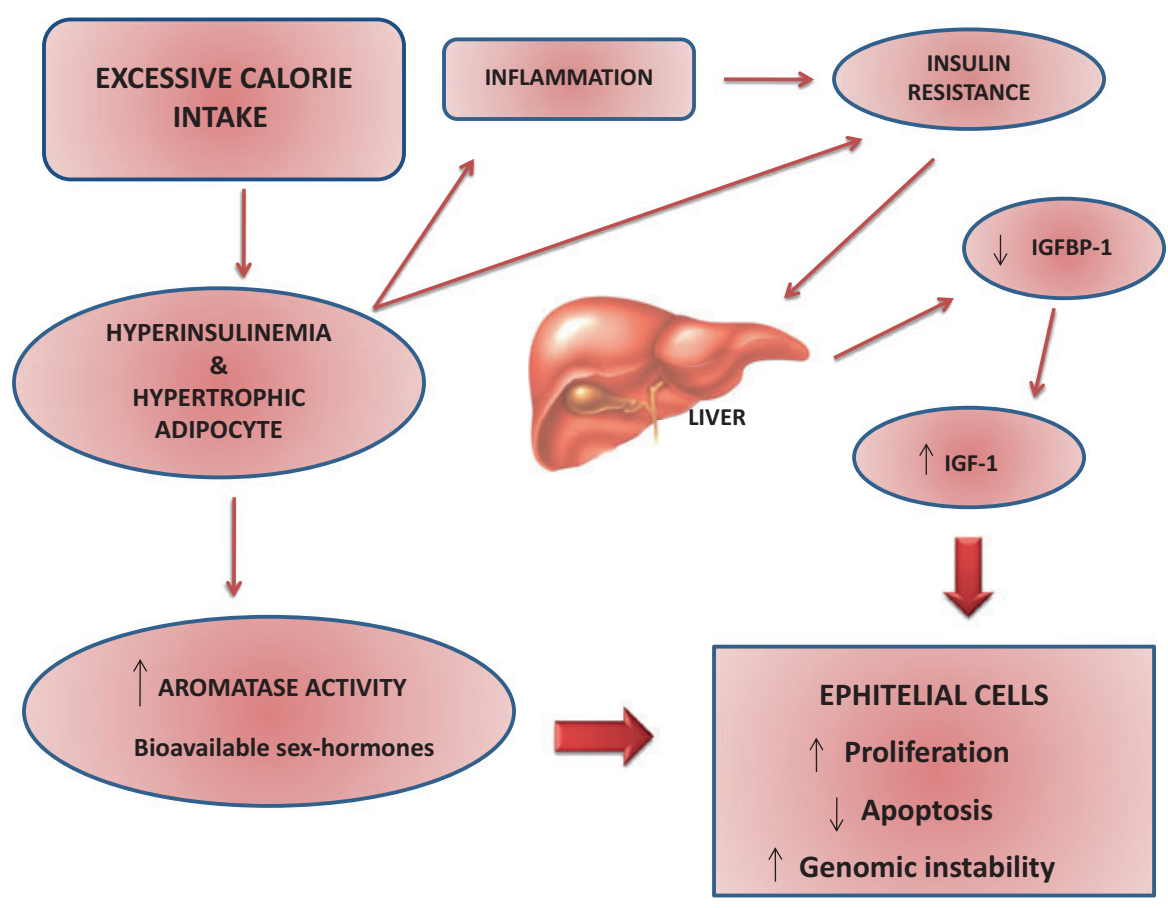

Fig. 3 Correlation between calorie intake and adiposity. Excessive calorie intake causes hyperinsulinemia, hypertrophy of adipose tissue, and increased inflammation

oxidative stress resistance (Madia et al. 2009; Hlavata et al. 2003). Additionally, Tor/ Sch9 and Ras/AC/PKA pathways regulate the expression of several DNA repair genes, including REV1 gene (Madia et al. 2009).

According to this evidence, Mn-superoxide dismutase (MnSOD) heterozygous knockout mice showed an increased DNA oxidative damage and tumor incidence. This suggests a complex interaction between oxidative stress and cancer (Van Remmen et al. 2003).

Another mechanism induced by DR is the autophagy response to oxidative stress. Autophagy is a process by which cells under DR conditions convey nutrients to essential metabolic processes. DR-induced autophagy is activated by poly (ADPribose) polymerase 1 (PARP-1), a nuclear enzyme induced by DNA damage. ROS (reactive oxygen species) production under DR conditions causes DNA damage, which determines PARP-1 activation and fasting-induced autophagy (Cangemi et al. 2016). ROS induce different types of DNA damage, including single-strand breaks (SSBs), double-strand breaks (DSBs), and ionized DNA nucleotides. The repair of latter damage requires the intervention of the base excision repair (BER) system, in particular the OGG1 (8-oxoguanine DNA glycosylase) enzyme 1. Both in vitro and in vivo experiments showed that BER activity is influenced by the availability of nutrients. Indeed, autophagy has no effects on OGG1 expression in the absence of fasting (Siggens et al. 2012). 
DEN-induced mice HCC cells exhibit high levels of Caspasi 3, PARP, and Citocromo $\mathrm{C}$, which are proteins involved in mitochondria-mediated autophagy, suggesting that DR suppresses proliferation and promotes apoptosis ( $\mathrm{Lu}$ et al. 2008; Duan et al. 2017).

According to previous studies (Xie et al. 2007; Standard et al. 2014), gene expression analysis of DEN-induced mice HCC cells revealed that DR restores functioning of many MAPK genes. MAPK pathway regulated by RAS promotes tumor growth and is one of the most important molecular targets for treatment of several types of cancers (Duan et al. 2017).

\section{The Implication of Dietary Restriction in Cancer}

The different molecular signature that distinguishes a normal cell from a tumor cell is the main reason that could explain the different susceptibility to growth stimuli. In fact, tumor cells undergo a series of genetic and epigenetic modifications that make their growth independent of the presence of growth factors (Hanahan and Weinberg 2011; Fig. 4). The accumulation of these genetic alterations constitutively activates key components of intracellular pathways. Among these, the most common deregulated signal pathways are Ras/Raf/MAPK and PTEN/PI3K/AKT, responsible for an uncontrolled cell proliferation (Massihnia et al. 2016). The deprivation of nutrients both in vitro and in vivo results in a decrease of growth factor levels in

Fig. 4 Growth stimuli of normal and malignant cells. The different molecular profile between normal and malignant cells is responsible for the differences in behavior toward growth stimuli

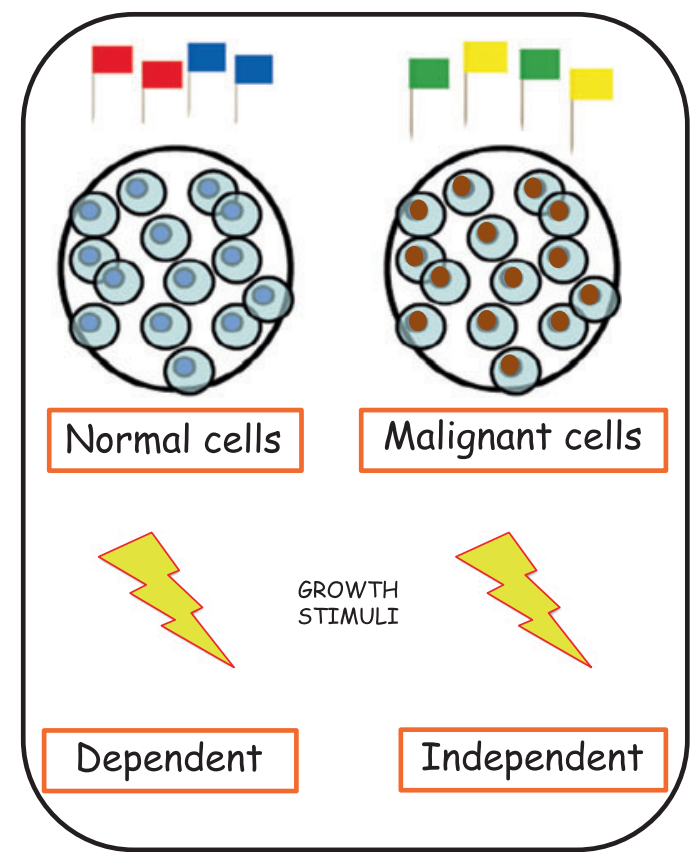


normal cells, forcing thus the cell to enter a proliferative quiescent status (Flemstrom et al. 2010; Pirkmajer and Chibalin 2011). Unlike normal cells, tumor cells overcome this block by reprogramming their metabolic state and thus maintaining high proliferative abilities (Hanahan and Weinberg 2011). The discovery that different types of dietary restrictions can protect normal cells from the most common side effects of chemotherapy has recently raised interest in its possible clinical application. Moreover, STS seems to protect not only healthy cells but also increase the sensitivity of various types of cancer to the therapy (Lee and Longo 2011). Indeed, fasting in combination with chemotherapy determines increased cytotoxic effects in malignant cells from different types of cancers (Russo and Rizzo 2008). The validity of STS has been evaluated in immunosuppressed nude mice xenograft models in which human neuroblastoma cells were subcutaneously injected. Surprisingly, after 34 days of fasting combined with cyclophosphamide treatment the tumor mass was reduced (Lee et al. 2012a). The usefulness of STS has also been demonstrated for its attenuating properties on chemotherapy side effects. Indeed, its cardioprotective properties have been recently demonstrated during doxorubicin-based treatments (Dirks-Naylor et al. 2014). In addition, a recent work on murine models revealed that following a prolonged fasting of $48-60 \mathrm{~h}$ prior to the administration of a high dose of etoposide, the side effects, generally resulting from the treatment, were attenuated (Raffaghello et al. 2008). The synergy between refeeding and DNA damage caused by pharmacological treatment may favor the growth of new foci in various organs including liver, colon, and rectum (Laconi et al. 1995; Premoselli et al. 1998). Interestingly, in a tumor mass, malignant cells are strictly connected with the socalled "cancer-associated adipocytes" (CAAs) and interact with them (Calle and Kaaks 2004). In particular, CAAs show the reduction of peculiar markers including HSL, APN, and resistin, and increased proinflammatory cytokine expression such as IL-6 and IL-1 $\beta$ and TNF- $\alpha$ (Berstein et al. 2007; Ribeiro et al. 2012; Dirat et al. 2011). This altered expression, associated with the production of adipokines, results in a tumor microenvironment variation that favors uncontrolled growth. Therefore, fasting, having a massive effect on the size of adipocytes, can consequently decrease the secretion of tumor-favorable factors (Hermsdorff et al. 2009). Recently, CR efficacy has also been demonstrated in relation to radiotherapy, leading to an increase in the sensitivity to radiation-induced cytotoxicity (Champ et al. 2013). As alternative to standard chemo-/radiotherapy, another type of metabolic therapy has been proposed (ketogenic diet) whose beneficial effects have been demonstrated in the multiform glioblastoma and brain cancer for its antiangiogenic, anti-inflammatory, and antiapoptotic abilities (Seyfried et al. 2015). Below we will discuss deeper the association of chemotherapy and dietary restriction in some of the most spread cancers worldwide looking at the benefits deriving from their combination (Fig. 5).

\section{Breast Cancer}

Breast cancer is one of the main causes of cancer deaths in the female population (Fanale et al. 2013). Various clinical studies have shown the efficacy of fasting in the 


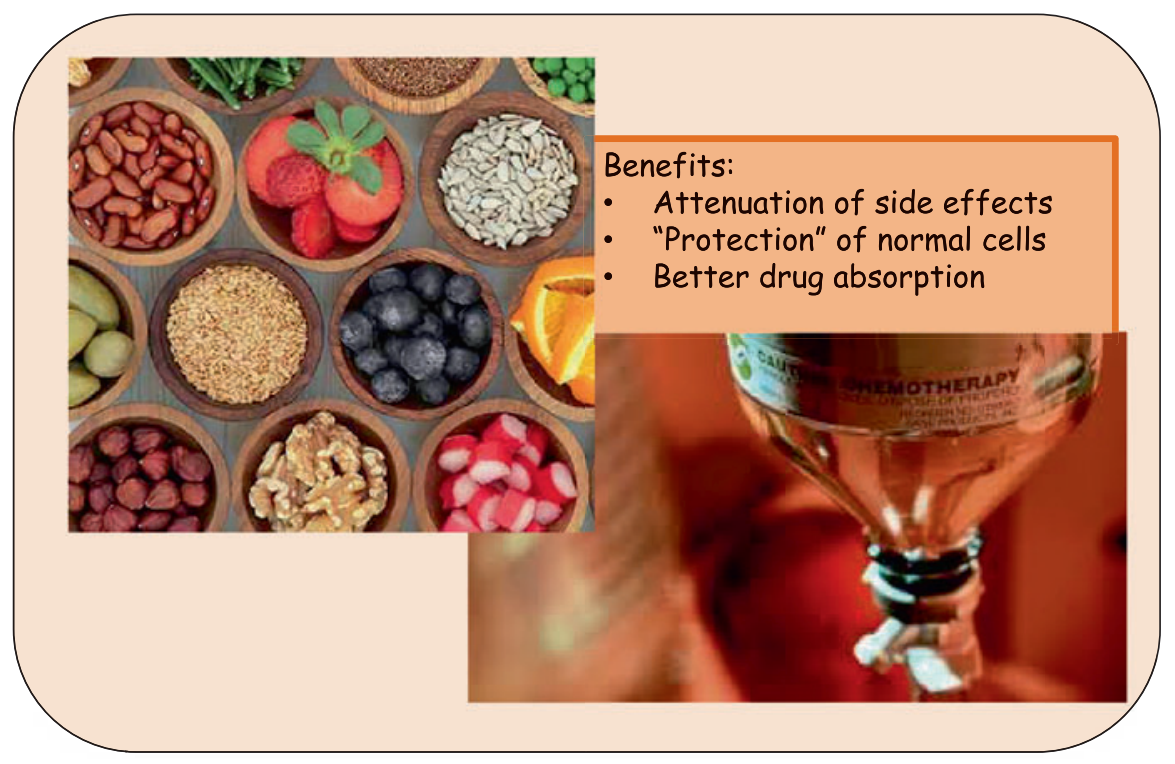

Fig. 5 Association between dietary restriction and chemotherapy. The figure shows the relevant benefits arising from the association between different types of dietary restriction and drug administration

favorable outcome of the chemotherapy treatment to which some patients affected by breast cancer have undergone. In particular, it seems that a short period of fasting pre- and posttreatment will have a better outcome in terms of patient's tolerability by reducing the side effects. Indeed, the case report of three different patients treated with different therapies and subjected to different times of fasting is below described. A first woman of 51 years with a breast cancer at stage $2 \mathrm{~A}$ did not show any side effect once subjected to fasting $140 \mathrm{~h}$ before and $40 \mathrm{~h}$ after treatment with docetaxel and cyclophosphamide. The validity of the association was confirmed in a second 53-year-old patient, also suffering of a tumor in stage 2A and HER2 +. In particular, chemotherapy cycles associated with fasting $64 \mathrm{~h}$ before and $24 \mathrm{~h}$ after were not accompanied by high toxicity effects or in any case with negligible and/or reversible transient effects. The third case saw a 78-year-old patient with a HER2+ tumor, after mastectomy and subjected to variable fasting periods in the course of carboplatinbased, docetaxel and trastuzumab-based chemotherapy cycles. Significant levels of pharmacological toxicity have not been reported (Safdie et al. 2009; Table 1).

\section{Ovarian Cancer}

Among gynecological tumors, ovarian cancer is one of the most common and the fifth cause of death in the female population (Reid et al. 2017). Al-Wahab et al. (2014) published a study showing the effects of energy balance in mouse models 
Table 1 Case reports of different tumors. The table summarizes the cases described in the text and related fasting schedules adopted in pre- and posttreatment

\begin{tabular}{|c|c|c|}
\hline \multirow[t]{3}{*}{ Breast cancer } & Case I (51yo) & $\begin{array}{l}140 \mathrm{~h} \text { before } \\
40 \mathrm{~h} \text { after treatment }\end{array}$ \\
\hline & Case II (53yo) & $\begin{array}{l}64 \mathrm{~h} \text { before } \\
24 \mathrm{~h} \text { after } \\
\text { Treatment }\end{array}$ \\
\hline & Case III (78yo) & Not shown \\
\hline Ovarian cancer & Case I (44yo) & $\begin{array}{l}62 \mathrm{~h} \text { before } \\
24 \mathrm{~h} \text { after treatment }\end{array}$ \\
\hline Lung cancer & Case I (61yo) & $\begin{array}{l}48 \mathrm{~h} \text { before } \\
24 \mathrm{~h} \text { after treatment }\end{array}$ \\
\hline Prostate cancer & Case I (74yo) & $\begin{array}{l}60 \mathrm{~h} \text { before } \\
24 \mathrm{~h} \text { after treatment }\end{array}$ \\
\hline
\end{tabular}

subjected to high energy diet or CR conditions. Mice group under high-energy diet showed the most extensive tumor formation accompanied by the highest tumor score at multiple sites. Moreover, they showed increased levels of insulin, leptin, IGF-1, VEGF, and proinflammatory factors (IL-6). Instead, the mice group under CR showed a lower tumor burden as well as a great reduction in insulin, IGF-1, leptin, MCP-1, VEGF, and IL-6 levels (Al-Wahab et al. 2014). Also, clinical trials demonstrated the effectiveness of the association chemotherapy/DR. The case of a 44-yearold woman suffering from ovarian cancer has been emblematic, because she has benefited from the antineoplastic treatment in combination with STS carried out $62 \mathrm{~h}$ before and extended $24 \mathrm{~h}$ after drug treatment (Safdie et al. 2009; Table 1).

\section{Lung Cancer}

Lung cancer is one of the major causes of cancer-related morbidity and death in men and women population worldwide. Depending on EGFR mutational status, therapy may vary in favor of tyrosine-kinase inhibitors (TKIs). Among them, erlotinib is one of the most commonly used TKIs in I and II line of treatment (Passiglia et al. 2017). Currently, the recommended dose is $150 \mathrm{mg}$ under complete fasting conditions or $2 \mathrm{~h}$ after the meal consumption. Two modalities of administration are resulted able to determine a different drug absorption and consequent increase in therapeutic efficacy. In particular, drug seems to have greater effect when the administration takes place $2 \mathrm{~h}$ after the meal (Katsuya et al. 2015). An interesting clinical case is that of a 61-year-old NSCLC patient who has seen mitigating the side effects of drug therapy after STS $48 \mathrm{~h}$ before and $24 \mathrm{~h}$ after therapy (Safdie et al. 2009; Table 1).

\section{Prostate Cancer}

Prostate cancer is considered as the second cause of cancer-related death in male population (Vanacore et al. 2017). Interesting is the case of a 74-year-old man 
diagnosed with stage 2 prostate adenocarcinoma. The patient has undergone several cycles of chemotherapy during which he faced many side effects such as fatigue, weakness, short-term memory impairment, and peripheral neuropathy. Moreover, PSA levels raised with an unstoppable trend. After many failed attempts, patients were enrolled in a rigorous fasting schedule consisting of restrictions $60 \mathrm{~h}$ prior to and $24 \mathrm{~h}$ post drug administration. After treatment, PSA levels dropped dramatically and a marked reduction of side effects was reported (Safdie et al. 2009; Table 1).

\section{Conclusion}

Recently, accumulating evidence showed that starvation condition seems to play a pivotal role in preventing cancer development and progression as well as improving the response to different therapeutic treatments. The above presented studies and considerations seem to confirm the protective role exerted by fasting against cancer. However, a potential limitation is represented by time required for each cancer patient to obtain an optimal fasting condition, since a severe nutrient deprivation for several months may be needed, as showed by in vivo preclinical analyses. Furthermore, not all patients are fit to undergo such dietary regimens, because many of them are subject to weight loss due to the chemotherapy toxicity and tumor itself. For this reason, since CR and ketogenic diet (KD) have been shown to be approaches particularly effective unlike intermittent fasting whose role is still controversial, further clinical studies are yet necessary in order to assess the safety and efficacy of these methods.

\section{Policies and Protocols}

\section{Protocol for Maintaining Cancer Cells Under Short-Term Starvation Conditions}

In this chapter, we have discussed the most significant studies present in literature concerning the molecular mechanisms by which dietary restriction may contribute to prevent cancer development, slow down its progression, and positively influence therapy response. Since most of the current experimental evidences concerning the correlation between dietary restriction and cancer arise from studies mainly performed on in vitro preclinical models, here we discuss a rapid and convenient method for maintaining the healthy and malignant breast cells under short-term starvation conditions restricting the supply of glucose. Glucose metabolism represents a primary source of energy able to support cell proliferation and regulate cell death-related signaling pathways. The impaired balance between excessively high glucose consumption and its poor supply determines glucose deprivation in the tumor microenvironment, activating a positive feedback mechanism that involves 
ROS production by NADPH oxidase and mitochondria, inhibition of tyrosine phosphatases by oxidation, and amplification of tyrosine kinase signaling in cells dependent on glucose for their survival.

Both cell lines are grown at $37{ }^{\circ} \mathrm{C}, 5 \% \mathrm{CO} 2$, and $80 \%$ confluence, in a culture medium DMEM (Dulbecco's Modified Eagle Medium) containing high glucose concentration (4.5 g/l D-glucose, $110 \mathrm{mM}$ pyruvate) and enriched with fetal bovine serum (10\% FBS), nonessential amino acids (NEAA-1\%), and streptomycin-penicillin ( $1 \% \mathrm{Strepto} / \mathrm{Pen})$. For short-term starvation experiments aimed at establishing a glucose deprivation, cells are washed twice with PBS (phosphate-buffered saline) and then incubated in glucose-free DMEM without pyruvate and supplemented with $10 \%$ FBS, $1 \%$ NEAA, $1 \%$ Strepto/Pen.

\section{Dictionary of Terms}

- Caloric restriction - Reduction of calorie intake that implicates feeding once daily or thrice weekly. It can be distinguished in two forms: intermittent caloric restriction and chronic caloric restriction.

- Dietary restriction - Condition of short- or long-term fasting which can involve the lack of food consumption for short or prolonged periods. It includes the caloric restriction, short- and long-term starvation, and ketogenic diet.

- Hyperinsulinemia - The increase in circulating insulin levels that lead to a greater IGF-1 activity, by reducing synthesis and secretion of IGF binding protein 1 , and elevated concentration of circulating sex hormones.

- Inflammatory response - Set of actions exerted by the immune system to fight an inflammation through release of several factors that mediate this response, including chemokines and cytokines.

- Therapy response - Assessment of the extent of sensitivity or resistance of a tumor to a specific anticancer treatment.

\section{Summary Points}

- This chapter focuses on molecular mechanisms by which dietary restriction may contribute to prevent cancer onset and improve therapy response.

- Dietary restriction involves food deprivation for short or prolonged periods.

- Dietary restriction includes short-term starvation, long-term starvation, caloric restriction, and ketogenic diet.

- Numerous experimental evidences showed that fasting may exert a protective role against aging and other age-related pathologies as well as cancer.

- Preclinical models suggested the potential use of fasting to induce anticancer effects and improve patient life's quality.

- However, prolonged fasting periods could impair the patient health conditions already unfavorable due to physiological weight loss induced by tumor itself. 
- Fasting-mediated benefits seem to be mediated by the reduction of inflammatory response and down-regulation of nutrient-related signaling pathways able to modulate cell proliferation and apoptosis.

- Good results were obtained in animal models by associating caloric restriction with conventional chemotherapies.

- Deregulation of IGF-1 and PI3K/AKT pathways causes dietary restriction resistance.

- This chapter describes some case reports for different types of cancer.

\section{References}

Al-Wahab Z, Tebbe C, Chhina J, Dar SA, Morris RT, Ali-Fehmi R, Giri S, Munkarah AR, Rattan R (2014) Dietary energy balance modulates ovarian cancer progression and metastasis. Oncotarget 5:6063-6075

Anton S, Leeuwenburgh C (2013) Fasting or caloric restriction for healthy aging. Exp Gerontol 48:1003-1005

Berstein LM, Kovalevskij AY, Poroshina TE, Kotov AV, Kovalenko IG, Tsyrlina EV, Leenman EE, Revskoy SY, Semiglazov VF, Pozharisski KM (2007) Signs of proinflammatory/genotoxic switch (adipogenotoxicosis) in mammary fat of breast cancer patients: role of menopausal status, estrogens and hyperglycemia. Int J Cancer 121:514-519

Brandhorst S, Longo VD (2016) Fasting and caloric restriction in cancer prevention and treatment. Recent Results Cancer Res 207:241-266

Brandhorst S, Harputlugil E, Mitchell JR, Longo VD (2017) Protective effects of short-term dietary restriction in surgical stress and chemotherapy. Ageing Res Rev. doi:10.1016/j.arr.2017.02.001

Cadoni E, Marongiu F, Fanti M, Serra M, Laconi E (2017) Caloric restriction delays early phases of carcinogenesis via effects on the tissue microenvironment. Oncotarget 8:36020

Calle EE, Kaaks R (2004) Overweight, obesity and cancer: epidemiological evidence and proposed mechanisms. Nat Rev Cancer 4:579-591

Cangemi A, Fanale D, Rinaldi G, Bazan V, Galvano A, Perez A, Barraco N, Massihnia D, Castiglia M, Vieni S, Bronte G, Mirisola M, Russo A (2016) Dietary restriction: could it be considered as speed bump on tumor progression road? Tumour Biol 37:7109-7118

Champ CE, Baserga R, Mishra MV, Jin L, Sotgia F, Lisanti MP, Pestell RG, Dicker AP, Simone NL (2013) Nutrient restriction and radiation therapy for cancer treatment: when less is more. Oncologist 18:97-103

Chiba T, Ezaki O (2010) Dietary restriction suppresses inflammation and delays the onset of stroke in stroke-prone spontaneously hypertensive rats. Biochem Biophys Res Commun 399:98-103

Cleary MP, Grossmann ME (2011) The manner in which calories are restricted impacts mammary tumor cancer prevention. J Carcinog 10:21

Cohen HY, Miller C, Bitterman KJ, Wall NR, Hekking B, Kessler B, Howitz KT, Gorospe M, De Cabo R, Sinclair DA (2004) Calorie restriction promotes mammalian cell survival by inducing the Sirt1 deacetylase. Science 305:390-392

Dirat B, Bochet L, Dabek M, Daviaud D, Dauvillier S, Majed B, Wang YY, Meulle A, Salles B, Le Gonidec S, Garrido I, Escourrou G, Valet P, Muller C (2011) Cancer-associated adipocytes exhibit an activated phenotype and contribute to breast cancer invasion. Cancer Res $71: 2455-2465$

Dirks-Naylor AJ, Kouzi SA, Yang S, Tran NT, Bero JD, Mabolo R, Phan DT, Whitt SD, Taylor HN (2014) Can short-term fasting protect against doxorubicin-induced cardiotoxicity? World J Biol Chem 5:269-274

Duan T, Sun W, Zhang M, Ge J, He Y, Zhang J, Zheng Y, Yang W, Shen HM, Yang J, Zhu X, Yu P (2017) Dietary restriction protects against diethylnitrosamine-induced hepatocellular tumorigenesis by restoring the disturbed gene expression profile. Sci Rep 7:43745 
Esposito K, Pontillo A, Di Palo C, Giugliano G, Masella M, Marfella R, Giugliano D (2003) Effect of weight loss and lifestyle changes on vascular inflammatory markers in obese women: a randomized trial. JAMA 289:1799-1804

Fanale D, Bazan V, Corsini LR, Caruso S, Insalaco L, Castiglia M, Cicero G, Bronte G, Russo A (2013) HIF-1 is involved in the negative regulation of AURKA expression in breast cancer cell lines under hypoxic conditions. Breast Cancer Res Treat 140:505-517

Fanale D, Amodeo V, Caruso S (2017) The interplay between metabolism, PPAR signaling pathway, and cancer. PPAR Res 2017:1-2

Flemstrom G, Bengtsson MW, Makela K, Herzig KH (2010) Effects of short-term food deprivation on orexin-A-induced intestinal bicarbonate secretion in comparison with related secretagogues. Acta Physiol (Oxf) 198:373-380

Fontana L, Weiss EP, Villareal DT, Klein S, Holloszy JO (2008) Long-term effects of calorie or protein restriction on serum IGF-1 and IGFBP-3 concentration in humans. Aging Cell 7:681-687

Giovannucci E, Pollak M, Liu Y, Platz EA, Majeed N, Rimm EB, Willett WC (2003) Nutritional predictors of insulin-like growth factor I and their relationships to cancer in men. Cancer Epidemiol Biomark Prev 12:84-89

Hanahan D, Weinberg RA (2011) Hallmarks of cancer: the next generation. Cell 144:646-674

Heilbronn LK, De Jonge L, Frisard MI, Delany JP, Larson-Meyer DE, Rood J, Nguyen T, Martin CK, Volaufova J, Most MM, Greenway FL, Smith SR, Deutsch WA, Williamson DA, Ravussin E, Pennington CT (2006) Effect of 6-month calorie restriction on biomarkers of longevity, metabolic adaptation, and oxidative stress in overweight individuals: a randomized controlled trial. JAMA 295:1539-1548

Hermsdorff HH, Zulet MA, Abete I, Martinez JA (2009) Discriminated benefits of a Mediterranean dietary pattern within a hypocaloric diet program on plasma RBP4 concentrations and other inflammatory markers in obese subjects. Endocrine 36:445-451

Hlavata L, Aguilaniu H, Pichova A, Nystrom T (2003) The oncogenic RAS2(val19) mutation locks respiration, independently of PKA, in a mode prone to generate ROS. EMBO J 22:3337-3345

Jin J, Iakova P, Jiang Y, Medrano EE, Timchenko NA (2011) The reduction of SIRT1 in livers of old mice leads to impaired body homeostasis and to inhibition of liver proliferation. Hepatology 54:989-998

Kalaany NY, Sabatini DM (2009) Tumours with PI3K activation are resistant to dietary restriction. Nature 458:725-731

Katsuya Y, Fujiwara Y, Sunami K, Utsumi H, Goto Y, Kanda S, Horinouchi H, Nokihara H, Yamamoto N, Takashima Y, Osawa S, Ohe Y, Tamura T, Hamada A (2015) Comparison of the pharmacokinetics of erlotinib administered in complete fasting and $2 \mathrm{~h}$ after a meal in patients with lung cancer. Cancer Chemother Pharmacol 76:125-132

Klement RJ, Fink MK (2016) Dietary and pharmacological modification of the insulin/IGF-1 system: exploiting the full repertoire against cancer. Oncogene 5:e193

La Paglia L, Listi A, Caruso S, Amodeo V, Passiglia F, Bazan V, Fanale D (2017) Potential role of ANGPTL4 in the cross talk between metabolism and cancer through PPAR signaling pathway. PPAR Res 2017:8187235

Laconi E, Tessitore L, Milia G, Yusuf A, Sarma DS, Todde P, Pani P (1995) The enhancing effect of fasting/refeeding on the growth of nodules selectable by the resistant hepatocyte model in rat liver. Carcinogenesis 16:1865-1869

Lee C, Longo VD (2011) Fasting vs dietary restriction in cellular protection and cancer treatment: from model organisms to patients. Oncogene 30:3305-3316

Lee C, Longo V (2016) Dietary restriction with and without caloric restriction for healthy aging. F1000Research 5:F1000 Faculty Rev-117. doi:10.12688/f1000research.7136.1

Lee C, Raffaghello L, Brandhorst S, Safdie FM, Bianchi G, Martin-Montalvo A, Pistoia V, Wei M, Hwang S, Merlino A, Emionite L, De Cabo R, Longo VD (2012a) Fasting cycles retard growth of tumors and sensitize a range of cancer cell types to chemotherapy. Sci Transl Med 4:124ra27 
Lee C, Raffaghello L, Longo VD (2012b) Starvation, detoxification, and multidrug resistance in cancer therapy. Drug Resist Updat 15:114-122

Lefevre M, Redman LM, Heilbronn LK, Smith JV, Martin CK, Rood JC, Greenway FL, Williamson DA, Smith SR, Ravussin E, Pennington CT (2009) Caloric restriction alone and with exercise improves CVD risk in healthy non-obese individuals. Atherosclerosis 203:206-213

Lluch GL, Lv M, Zhu X, Wang H, Wang F, Guan W (2014) Roles of caloric restriction, Ketogenic diet and intermittent fasting during initiation, progression and metastasis of cancer in animal models: a systematic review and meta-analysis. PLoS One 9:e115147

Longo VD, Fontana L (2010) Calorie restriction and cancer prevention: metabolic and molecular mechanisms. Trends Pharmacol Sci 31:89-98

Longo VD, Mattson MP (2014) Fasting: molecular mechanisms and clinical applications. Cell Metab 19:181-192

Longo VD, Antebi A, Bartke A, Barzilai N, Brown-Borg HM, Caruso C, Curiel TJ, De Cabo R, Franceschi C, Gems D, Ingram DK, Johnson TE, Kennedy BK, Kenyon C, Klein S, Kopchick JJ, Lepperdinger G, Madeo F, Mirisola MG, Mitchell JR, Passarino G, Rudolph KL, Sedivy JM, Shadel GS, Sinclair DA, Spindler SR, Suh Y, Vijg J, Vinciguerra M, Fontana L (2015) Interventions to slow aging in humans: are we ready? Aging Cell 14:497-510

Lu C, Shi Y, Wang Z, Song Z, Zhu M, Cai Q, Chen T (2008) Serum starvation induces H2AX phosphorylation to regulate apoptosis via p38 MAPK pathway. FEBS Lett 582:2703-2708

Lv M, Zhu X, Wang H, Wang F, Guan W (2014) Roles of caloric restriction, ketogenic diet and intermittent fasting during initiation, progression and metastasis of cancer in animal models: a systematic review and meta-analysis. PLoS One 9:e115147

Madia F, Wei M, Yuan V, Hu J, Gattazzo C, Pham P, Goodman MF, Longo VD (2009) Oncogene homologue Sch9 promotes age-dependent mutations by a superoxide and Rev1/Polzeta-dependent mechanism. J Cell Biol 186:509-523

Massihnia D, Galvano A, Fanale D, Perez A, Castiglia M, Incorvaia L, Listi A, Rizzo S, Cicero G, Bazan V, Castorina S, Russo A (2016) Triple negative breast cancer: shedding light onto the role of pi3k/akt/mtor pathway. Oncotarget 7:60712-60722

Passiglia F, Listi A, Castiglia M, Perez A, Rizzo S, Bazan V, RUSSO A (2017) EGFR inhibition in NSCLC: new findings. ... and opened questions? Crit Rev Oncol Hematol 112:126-135

Pirkmajer S, Chibalin AV (2011) Serum starvation: caveat emptor. Am J Physiol Cell Physiol 301: C272-C279

Premoselli F, Sesca E, Binasco V, Caderni G, Tessitore L (1998) Fasting/re-feeding before initiation enhances the growth of aberrant crypt foci induced by azoxymethane in rat colon and rectum. Int J Cancer 77:286-294

Prisco M, Romano G, Peruzzi F, Valentinis B, Baserga R (1999) Insulin and IGF-I receptors signaling in protection from apoptosis. Horm Metab Res 31:80-89

Raffaghello L, Lee C, Safdie FM, Wei M, Madia F, Bianchi G, Longo VD (2008) Starvationdependent differential stress resistance protects normal but not cancer cells against high-dose chemotherapy. Proc Natl Acad Sci U S A 105:8215-8220

Ramsey MM, Ingram RL, Cashion AB, Ng AH, Cline JM, Parlow AF, Sonntag WE (2002) Growth hormone-deficient dwarf animals are resistant to dimethylbenzanthracine (DMBA)-induced mammary carcinogenesis. Endocrinology 143:4139-4142

Reid BM, Permuth JB, Sellers TA (2017) Epidemiology of ovarian cancer: a review. Cancer Biol Med 14:9-32

Renehan AG, Zwahlen M, Minder C, O’Dwyer ST, Shalet SM, Egger M (2004) Insulin-like growth factor (IGF)-I, IGF binding protein-3, and cancer risk: systematic review and meta-regression analysis. Lancet 363:1346-1353

Ribeiro R, Monteiro C, Catalan V, Hu P, Cunha V, Rodriguez A, Gomez-Ambrosi J, Fraga A, Principe P, Lobato C, Lobo F, Morais A, Silva V, Sanches-Magalhaes J, Oliveira J, Pina F, Lopes C, Medeiros R, Fruhbeck G (2012) Obesity and prostate cancer: gene expression signature of human periprostatic adipose tissue. BMC Med 10:108

Robertson LT, Mitchell JR (2013) Benefits of short-term dietary restriction in mammals. Exp Gerontol 48:1043-1048 
Russo A, Rizzo S (2008) Could starvation minimize chemotherapy-induced toxicities? Expert Opin Ther Targets 12:1205-1207

Safdie FM, Dorff T, Quinn D, Fontana L, Wei M, Lee C, Cohen P, Longo VD (2009) Fasting and cancer treatment in humans: a case series report. Aging (Albany NY) 1:988-1007

Seyfried TN, Flores R, Poff AM, D'agostino DP, Mukherjee P (2015) Metabolic therapy: a new paradigm for managing malignant brain cancer. Cancer Lett 356:289-300

Shi Y, Felley-Bosco E, Marti TM, Orlowski K, Pruschy M, Stahel RA (2012) Starvation-induced activation of ATM/Chk2/p53 signaling sensitizes cancer cells to cisplatin. BMC Cancer 12:571

Siggens L, Figg N, Bennett M, Foo R (2012) Nutrient deprivation regulates DNA damage repair in cardiomyocytes via loss of the base-excision repair enzyme OGG1. FASEB J 26:2117-2124

Standard J, Jiang Y, Yu M, Su X, Zhao Z, Xu J, Chen J, King B, Lu L, Tomich J, Baybutt R, Wang W (2014) Reduced signaling of PI3K-Akt and RAS-MAPK pathways is the key target for weight-loss-induced cancer prevention by dietary calorie restriction and/or physical activity. J Nutr Biochem 25:1317-1323

Tinkum KL, Stemler KM, White LS, Loza AJ, Jeter-Jones S, Michalski BM, Kuzmicki C, Pless R, Stappenbeck TS, Piwnica-Worms D, Piwnica-Worms H (2015) Fasting protects mice from lethal DNA damage by promoting small intestinal epithelial stem cell survival. Proc Natl Acad Sci U S A 112:E7148-E7154

Toren P, Mora BC, Venkateswaran V (2013) Diet, obesity, and cancer progression: are adipocytes the link? Lipid Insights 6:37-45

Trepanowski JF, Canale RE, Marshall KE, Kabir MM, Bloomer RJ (2011) Impact of caloric and dietary restriction regimens on markers of health and longevity in humans and animals: a summary of available findings. Nutr J 10:107

Van Remmen H, Ikeno Y, Hamilton M, Pahlavani M, Wolf N, Thorpe SR, Alderson NL, Baynes JW, Epstein CJ, Huang TT, Nelson J, Strong R, Richardson A (2003) Life-long reduction in MnSOD activity results in increased DNA damage and higher incidence of cancer but does not accelerate aging. Physiol Genomics 16:29-37

Vanacore D, Boccellino M, Rossetti S, Cavaliere C, D'aniello C, Di Franco R, Romano FJ, Montanari M, La Mantia E, Piscitelli R, Nocerino F, Cappuccio F, Grimaldi G, Izzo A, Castaldo L, Pepe MF, Malzone MG, Iovane G, Ametrano G, Stiuso P, Quagliuolo L, Barberio D, Perdona S, Muto P, Montella M, Maiolino P, Veneziani BM, Botti G, Caraglia M, Facchini G (2017) Micrornas in prostate cancer: an overview. Oncotarget. doi:10.18632/oncot arget.16933

Wang YY, Lehuede C, Laurent V, Dirat B, Dauvillier S, Bochet L, Le Gonidec S, Escourrou G, Valet P, Muller C (2012) Adipose tissue and breast epithelial cells: a dangerous dynamic duo in breast cancer. Cancer Lett 324:142-151

Xie L, Jiang Y, Ouyang P, Chen J, Doan H, Herndon B, Sylvester JE, Zhang K, Molteni A, Reichle M, Zhang R, Haub MD, Baybutt RC, Wang W (2007) Effects of dietary calorie restriction or exercise on the PI3K and Ras signaling pathways in the skin of mice. J Biol Chem 282:28025-28035

Yamaza H, Komatsu T, Wakita S, Kijogi C, Park S, Hayashi H, Chiba T, Mori R, Furuyama T, Mori N, Shimokawa I (2010) FoxO1 is involved in the antineoplastic effect of calorie restriction. Aging Cell 9:372-382 